\title{
Beam Steerable Subarray with Small Footprint for Use as Building Block in Wall- Mounted Indoor 5G Infrastructure
}

\author{
Marzieh SalarRahimi ${ }^{1 *}$, Guy A. E. Vandenbosch ${ }^{1}$ \\ ${ }^{1}$ TELEMIC research division, Department of Electrical Engineering, KU Leuven, 3001 Leuven, Belgium \\ *marzieh.salarrahimi@kuleuven.be
}

\begin{abstract}
This paper is a preprint of a paper accepted by IET Microwaves, Antennas \& Propagation and is subject to Institution of Engineering and Technology Copyright. When the final version is published, the copy of record will be available at IET Digital Library
\end{abstract}




\title{
Beam steerable subarray with small footprint for use as building block in wall-mounted indoor wireless infrastructure
}

ISSN 1751-8725

Received on 28th June 2018 Accepted on 8th January 2019 doi: 10.1049/iet-map.2018.6189 www.ietdl.org

\author{
Marzieh SalarRahimi ${ }^{1}$, Guy A.E. Vandenbosch ${ }^{1}$ \\ ${ }^{1}$ TELEMIC Research Division, Department of Electrical Engineering, KU Leuven, 3001 Leuven, Belgium \\ $凶$ E-mail: marzieh.salarrahimi@kuleuven.be
}

\begin{abstract}
A dedicated antenna topology targeting wall-mounted beam-steered mass-producable wireless indoor applications in the microwave band is presented. Four linearly polarised $\mathrm{E}$-shaped microstrip patches are fed by a novel Butler matrix in a $2 \times 2$ array configuration, realising a very simple two-dimensional beam steering. To isolate the beam forming network (BFN) from the patches and the wall, a multilayer stripline technology was used. The proposed passive BFN consists of stripline couplers without crossings or physically separate phase shifters, leading to a BFN fitting within the same footprint as the array itself. The overall size of the Butler matrix is only $0.5410 \times 0.5410 \times 0.0610$. A prototype at $6 \mathrm{GHz}$ has been realised and measured, proving the validity of the concept.
\end{abstract}

\section{Introduction}

In future, one can envisage rooms, even complete houses/offices where the walls are covered with a dense network of antenna elements, forming a sparse-array topology. These antennas can even be applied to the walls by implementing them on a surface socalled 'e-wall-paper'. This infrastructure should be simple, targeting cheap mass-produced wall-mounted future indoor telecom applications.

There are several requirements that are believed to be crucial for such an infrastructure. First of all, it would be highly beneficiary if this future infrastructure could be deployed in a modular way, using a subarray with a small footprint (including the feeding circuits) as a building block to construct medium sized or larger antenna arrays where appropriate [1]. Second, the antennas need to be beam steerable, preferable in the whole hemispheric two-dimensional (2D) space of possible directions [2], this to optimise the link budget and to minimise interference. Third, the antennas, including the feeding networks, need to be electromagnetically decoupled from the walls, since the type of wall can be quite diverse. Fourth, since mass deployment is targeted, the subarray should be as cheap as possible.

Beam steering: There is a lot of literature that proposes beam forming networks (BFNs). Beam steering can be implemented in an analogue or a digital way [3-5]. Due to its specific structure, which has been thoroughly discussed in [6], a Butler matrix is a conventional analogue systematic method to drive antenna arrays [7-11]. It is well-established to drive linear arrays realising onedimensionally beam switching antennas. However, as mentioned in $[12,13]$, a Butler matrix can also drive 2D arrays. Several attempts have been made to design BFNs dedicated to this goal. The authors of [2] introduce a new Butler matrix configuration, making use of SIW directional couplers to excite a 2 by 2 antenna array. Also, a non-conventional BFN has been reported in [14]. However, the resulting structures are larger than a wavelength being unfit to be used as building block in larger arrays. In this work, a new modified Buttler matrix configuration is used to realise the beam steering.

Footprint: In general, a Butler matrix uses a network consisting of hybrids, phase shifters and crossovers. The authors of [15] replace the 90 -degree couplers and 45 -degree phase shifters by 45 -degree couplers. In this way, the phase shifters are eliminated, and the total phase tolerance of the matrix is improved. Some efforts also have been reported to remove crossovers in the network; resulting in simple designs as reported in [2, 16-18]. However, these proposed BFNs use long transmission lines and delay lines as 45degree phase shifters which increase the size and decrease the network efficiency $[16,19]$. Of course, it should be mentioned that crossovers, which introduce additional loss and mismatch, were also eliminated in [20]. However, there half-wavelength open stubs are used as phase shifters. The resulting structure is also quite large. Using left-handed transmission lines is also a way to miniaturise BFNs. This technique was used in [21]. However, the resulted structure still is not small enough. In this work, the Buttler matrix consists of only simple stripline couplers and short transmission lines. Physically separate phase shifters and crossovers are totally avoided. This lessening of the number of modules not only leads to lower phase and amplitude tolerances, but also to a very compact size.

Decoupling from wall: It has to be emphasised that the use of stripline technology is really crucial in our design, because it not only isolates the BFN from the wall and the antenna elements, but it also decreases the lateral size of the whole structure.

The way, in which the Butler matrix and the array are conceived and combined, specifically targeting wall-mounted beam-steered applications is the main innovation of this paper. The topology has all the necessary characteristics: the design is modular, very compact with small footprint, isolated from the wall, and on top simple and cost efficient, as shown in Fig. 1. A prototype has been realised in multilayer technology with Rogers substrates.

In the following sections, we will start with the concept of the new Butler matrix and BFN, continue with the array design, and finally discuss the prototype.

\section{Butler matrix}

\subsection{Concept}

The conceptual scheme of the proposed Butler matrix is shown in Fig. 2. The proposed Butler matrix provides phase relations just as conventional designs, but it just is composed of hybrids with 45degree and 90-degree phase differences in the first and last stages, respectively. The phase relations of a general Butler matrix are listed in Table 1 [15].

It is important to stress that the hybrids are straightforwardly connected to each other with short transmission lines, and in a particular arrangement. Obviously, the prominent advantage of this is its compactness compared to conventional schemes. In addition, a reduction of the destructive effect of mismatch losses is a worthwhile consequence of the concept. In other words, lower 


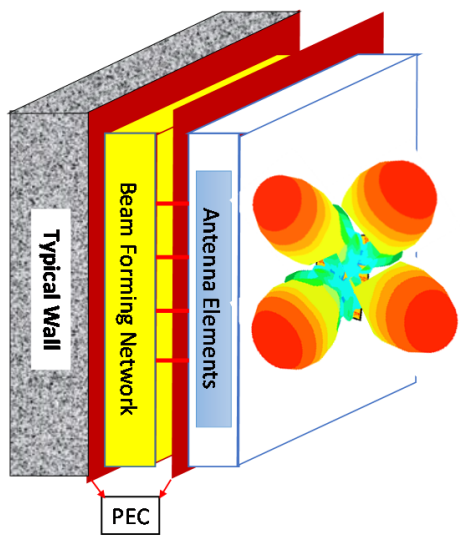

Fig. 1 Definition of problem
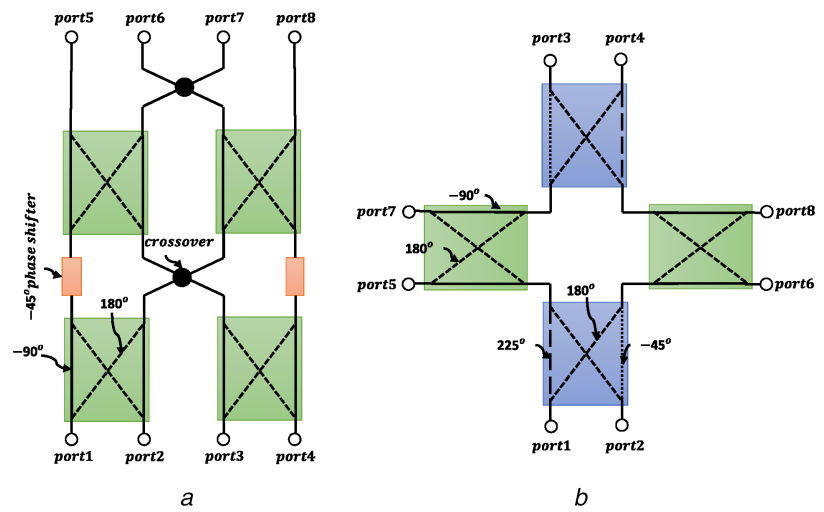

Fig. 2 Conceptual scheme of Butler matrix

(a) Conventional, (b) Proposed

Table 1 Phase relations of Butler matrix

\begin{tabular}{lccccc}
\hline Port & Port 5 & Port 6 & Port 7 & Port 8 & $\Delta \theta$ \\
\hline 1 & $135^{\circ}$ & $90^{\circ}$ & $45^{\circ}$ & $0^{\circ}$ & $45^{\circ}$ \\
2 & $90^{\circ}$ & $-135^{\circ}$ & $0^{\circ}$ & $135^{\circ}$ & $-135^{\circ}$ \\
3 & $135^{\circ}$ & $0^{\circ}$ & $-135^{\circ}$ & $90^{\circ}$ & $135^{\circ}$ \\
4 & $0^{\circ}$ & $45^{\circ}$ & $90^{\circ}$ & $135^{\circ}$ & $-45^{\circ}$ \\
\hline
\end{tabular}

phase and amplitude tolerances are achieved by lessening the number of components.

The general idea to design the couplers has been grasped from [22], where the inductive loading effect of crossed slots results in a significant size reduction. The topology of the couplers can be found in Fig. 3. The rationale behind the novel stripline coupler is the following. A resonant structure divides the input power between two output ports (hereinafter, half-power outputs) with an equal amplitude and with the correct phase difference. This is done by putting two asymmetrically loaded slots to divert the surface currents on the patch. The couplers are developed in stripline technology, in order to improve the network's isolation from surroundings.

\subsection{Design}

An actual design was made for operation around $6 \mathrm{GHz}$. The used design tool was CST Microwave Studio. The design is based on two Rogers Duroid RT5880 grounded substrates with a relative permittivity of 2.2 and 62 Mil of thickness, connected face to face.

The proposed BFN, that consists of 45-degree and 90-degree couplers, which are connected by short strip transmission lines, is shown in Fig. 3. Specifications of the couplers are presented.

The geometrical dimensions of the couplers along with their simulated responses are depicted in detail in Figs. 4 and 5. The inputs/outputs are $50 \Omega$ striplines. Note the matching transitions that are added between the $50 \Omega$ transmission lines and the squarepatch and the triangular cutting of some corners. Fig. 4 shows the

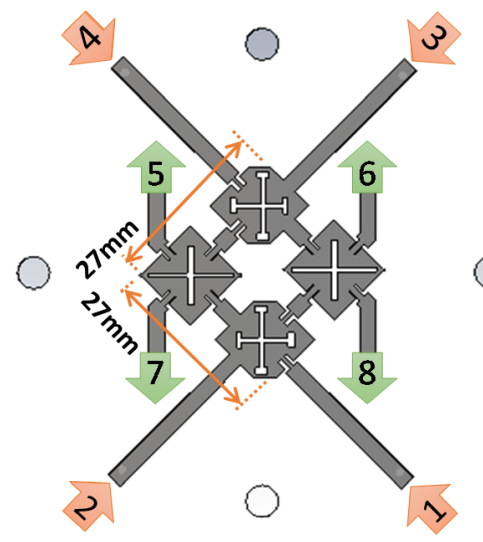

Fig. 3 Proposed Butler matrix
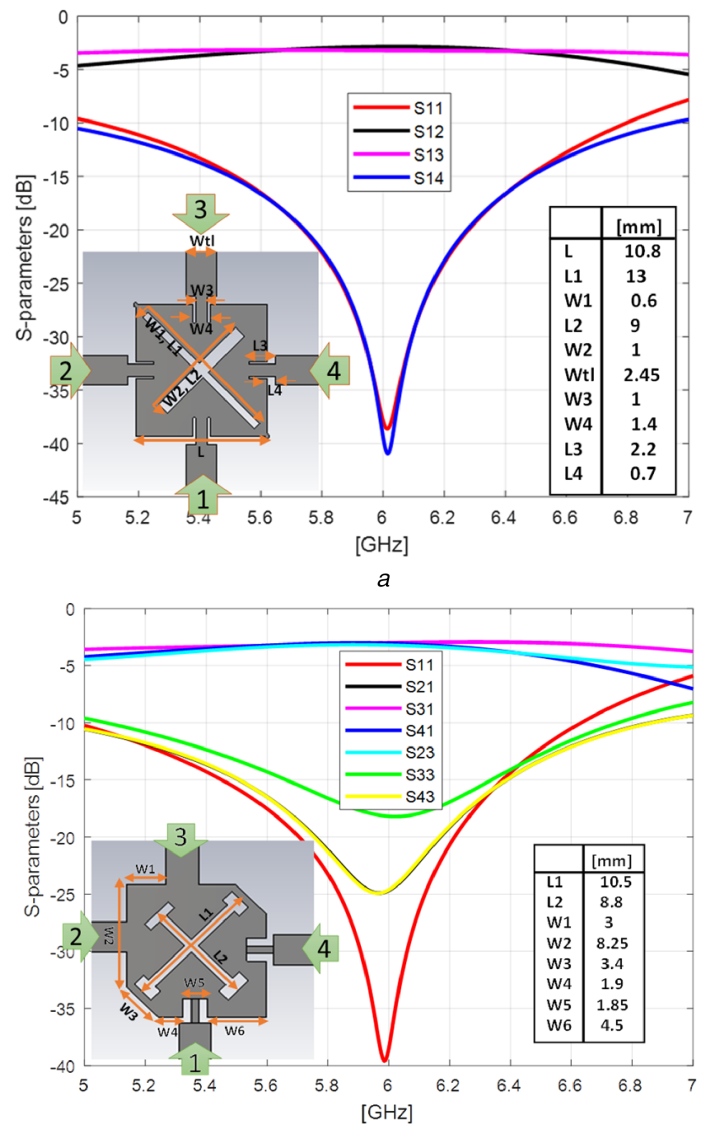

Fig. 4 Reflection and transmission coefficients of stripline couplers (a) 90-degree, (b) 45-degree

amplitude of the S-parameters for both couplers. Because of geometrical symmetry, just some of them are represented. As expected, the input power is divided between two half-power output ports with the correct phase difference. Fig. 5 shows the phase differences between the half-power output ports. For example, the black curve in Fig. 5 represents the phase difference between the half-power output ports 3 and 4, when port 1 of the 45-degree coupler is driven. In the desired frequency band, it has an acceptable deviation from the ideal value of $-45^{\circ}$.

The 90-degree coupler was designed based on [22]. W3, W4, L3 and $L 4$ were tuned to optimise input matching and isolation ( $S 11$ and $S 14$ ) at the centre frequency. The desired phase differences between the ports of the 45-degree coupler are controlled by two parameters; (i) an offset between the head-on ports, and (ii) a slant displacement of the shorter dumbbell slot with respect to the longer one. The 45-degree coupler was optimised to achieve two goals at the centre frequency: (i) keeping a $-45^{\circ}$ and a $225^{\circ}$ phase difference between the half-power outputs when ports 1 and 3 are 


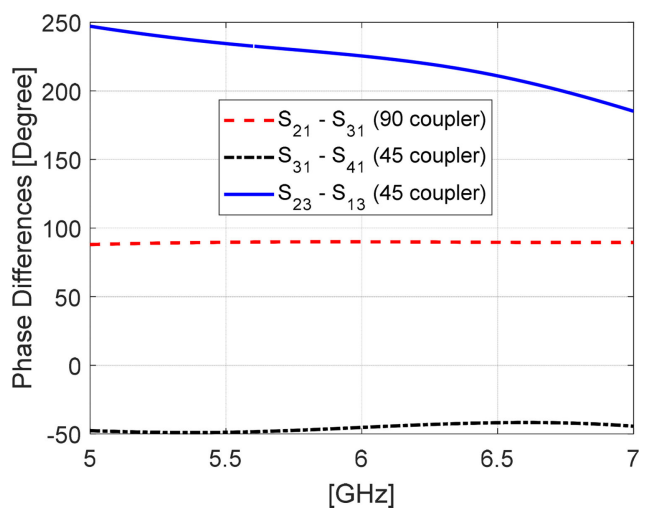

Fig. 5 Phase relations between ports of both stripline couplers
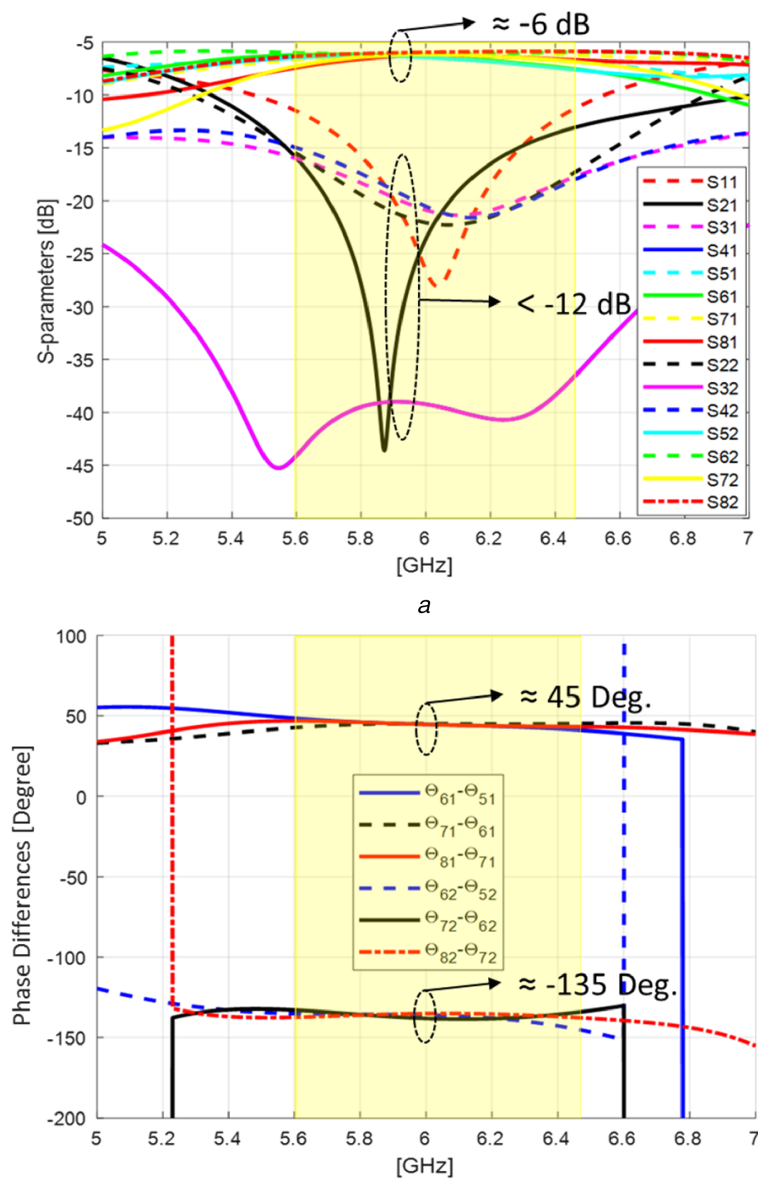

Fig. 6 Simulated results of the proposed BFN (a) Reflection and transmission coefficients, (b) Phase differences between output ports excited, respectively; and (ii) minimising the amplitudes of $S 11$, $S 33, S 21$, and $S 43$ to achieve optimised input matching and isolation.

A combination of the couplers and the connecting transmission lines results in the proposed $\mathrm{BFN}$, as shown in Fig. 3. The simulated amplitude and phase relations between the ports of the proposed BFN are shown in Fig. 6. The structure is fully symmetric. Ports 1 and 2 are equivalent to ports 4 and 3 , respectively. The proposed BFN operates in the $5.6-6.45 \mathrm{GHz}$ frequency band. Amplitude and phase tolerances with respect to nominal quantities are $<1.2 \mathrm{~dB}$ and $3^{\circ}$. The physical size is just $0.54 \lambda_{0} \times 0.54 \lambda_{0} \times 0.06 \lambda_{0}$, which makes it one of the smallest in the literature, as shown in Table 2. Note that although using lumped components may reduce the size even further, see [23, 24], these components often exhibit non-ideal parasitic effects that make them very hard to use at higher frequencies, and typically result in a more expensive structure. In addition, the resulting structure is typically not frequency-scalable. Table 2 compares the structures that do not suffer from these disadvantages. It has to be emphasised that Table 2 compares Butler matrices, not the arrays driven by them. All these Butler matrices can be used to drive linear or $2 \mathrm{D}$ arrays.

Note that the phase differences between the output ports of the BFN are sufficiently stable (Fig. $6 b$ ). On the other hand, the phase difference between ports 3 and 4 of the modified coupler are quite stable when port 1 is fed (black curve in Fig. 5), but this phase difference varies $\pm 10.2^{\circ}$ when port 2 is fed (blue curve in Fig. 5). Therefore, at a first glance, it is expected to see a quite flat curve and an unstable curve in Fig. $6 b$. However, both blue curves vary only ca. $\pm 3^{\circ}$ with moderately descending slopes. This can be explained by the fact that the phase differences between the ports of the couplers compensate each other.

\section{$32 \mathrm{D}$ array}

\subsection{Beam steering}

In this section, first we study the beams produced when a 2 by 2 array is driven by a general Butler matrix. The elements are placed on the corners of a square with size $D$. Fig. 7 shows the elements in the same-polarised and opposite-polarised arrangements. The elements are connected to ports 5-8 of a standard Butler matrix. Fig. $8 b$ represents the calculated radiation patterns for the samepolarised array, for $D$ equal to $0.5 \lambda_{0}$. The plotted radiation patterns are array factors multiplied with a unidirectional typical cosine element pattern. As Fig. $8 a$ shows, four directional beams are produced, depending on the selected input port of the BFN. These beams are tilted in elevation angle and each of them targets a particular azimuth angle, as illustrated in Fig. $8 b$. The radiation patterns are plotted in two azimuth planes, (i) the azimuth plane containing the main beam direction, and (ii) the azimuth plane normal to that. The array performance for $D$ equal to half a wavelength is acceptable. The side lobe levels are less than -13 $\mathrm{dB}$. It was observed that any increase in $D$ has a negative effect on SLL.

Table 2 Comparison with other reported 4 by 4 Butler matrix structures

\begin{tabular}{lccccc}
\hline Ref. & Size $\lambda_{0} \times \lambda_{0} \times \lambda_{0}$ & Band width, \% & $F_{0,} \mathrm{GHz}$ & Remarks & Technology \\
\hline$[2]$ & $2.86 \times 2.86 \times 0.220$ & 19.6 & 25.5 & phase shifter's insertion loss 1.26 dB & multilayer SIW \\
{$[9]$} & $11.0 \times 4.25 \times 0.050$ & 13.3 & 30 & - & single layer SIW \\
{$[12]$} & $1.23 \times 0.55 \times 0.012$ & 1.25 & 2.4 & - & single layer PCB \\
{$[13]$} & $2.97 \times 2.97 \times 0.015$ & 35.3 & 5.95 & - & single layer PCB \\
{$[21]$} & $0.97 \times 0.73 \times 0.004$ & $\mathrm{~N} . \mathrm{A}$. & 1.8 & - & single layer PCB \\
{$[15]$} & $1.14 \times 1.08 \times 0.016$ & 20.1 & 6 & - & single layer PCB \\
{$[16]$} & $4.80 \times 4.80 \times 0.003$ & 7.5 & 41.5 & - & single layer PCB \\
{$[20]$} & $1.73 \times 1.65 \times 0.020$ & 23.8 & 4 & single layer PCB \\
{$[19]$} & $9.28 \times 9.28 \times 0.050$ & 13.3 & 30 & - & radiation efficiency 46\% \\
- & $0.54 \times 0.54 \times 0.060$ & 14.1 & 6 & - & single layer SIW \\
this work & & - & - & multilayer PCB \\
\hline
\end{tabular}



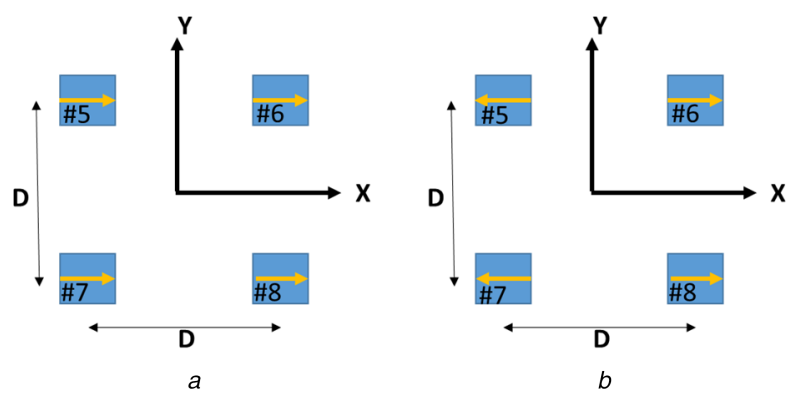

Fig. 7 Array of patch elements

(a) Same-polarised, (b) Opposite-polarised
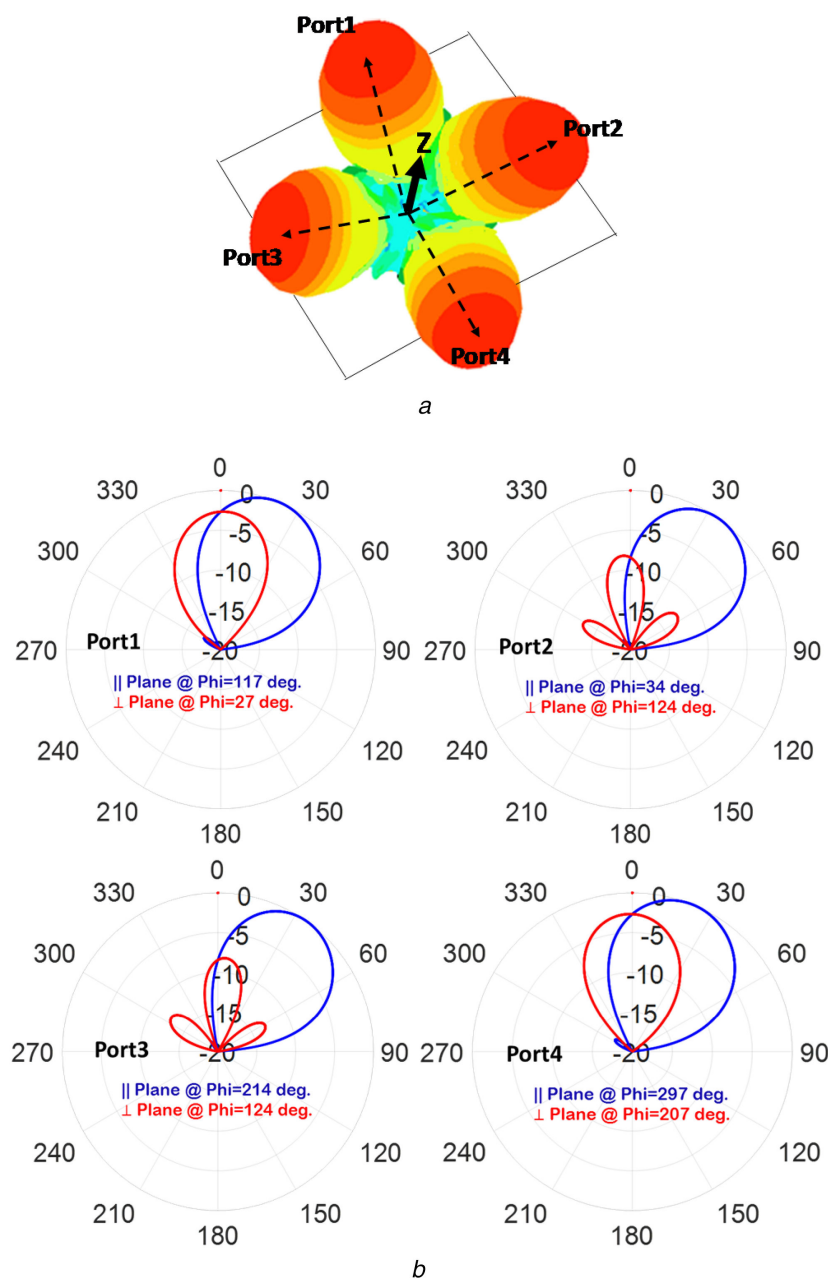

Fig. 8 Radiation pattern of the array driven by the Butler matrix, when D $=0.5 \lambda_{0}$

(a) 3D patterns for each of the four feeding ports superposed in one figure, (b) 2D patterns

Note that the opposite-polarised array configuration is used, taking advantage of the symmetrical geometry.

\subsection{Antenna design}

A 2 by 2 array prototype consisting of four E-shaped patch elements driven by the proposed BFN was designed. The E-shaped patch has been chosen because of its moderately wide bandwidth obtained with a single dielectric layer [25]. The patches are printed on a Rogers Duroid RT5870 grounded substrate with a relative permittivity of 2.33 and 60 Mil of thickness. The patches were connected to output ports of the BFN by vertical vias. The different layers and the assembled structure can be seen in Fig. 9.

The measured and simulated S-parameters of the four-port structure are depicted in Fig. 10. The $10 \mathrm{~dB}$ return loss/isolation bandwidth is from 5.9 to $6.13 \mathrm{GHz}$, i.e. $213 \mathrm{MHz}$. Taking into account the conclusions given in the benchmarking papers [26, 27],

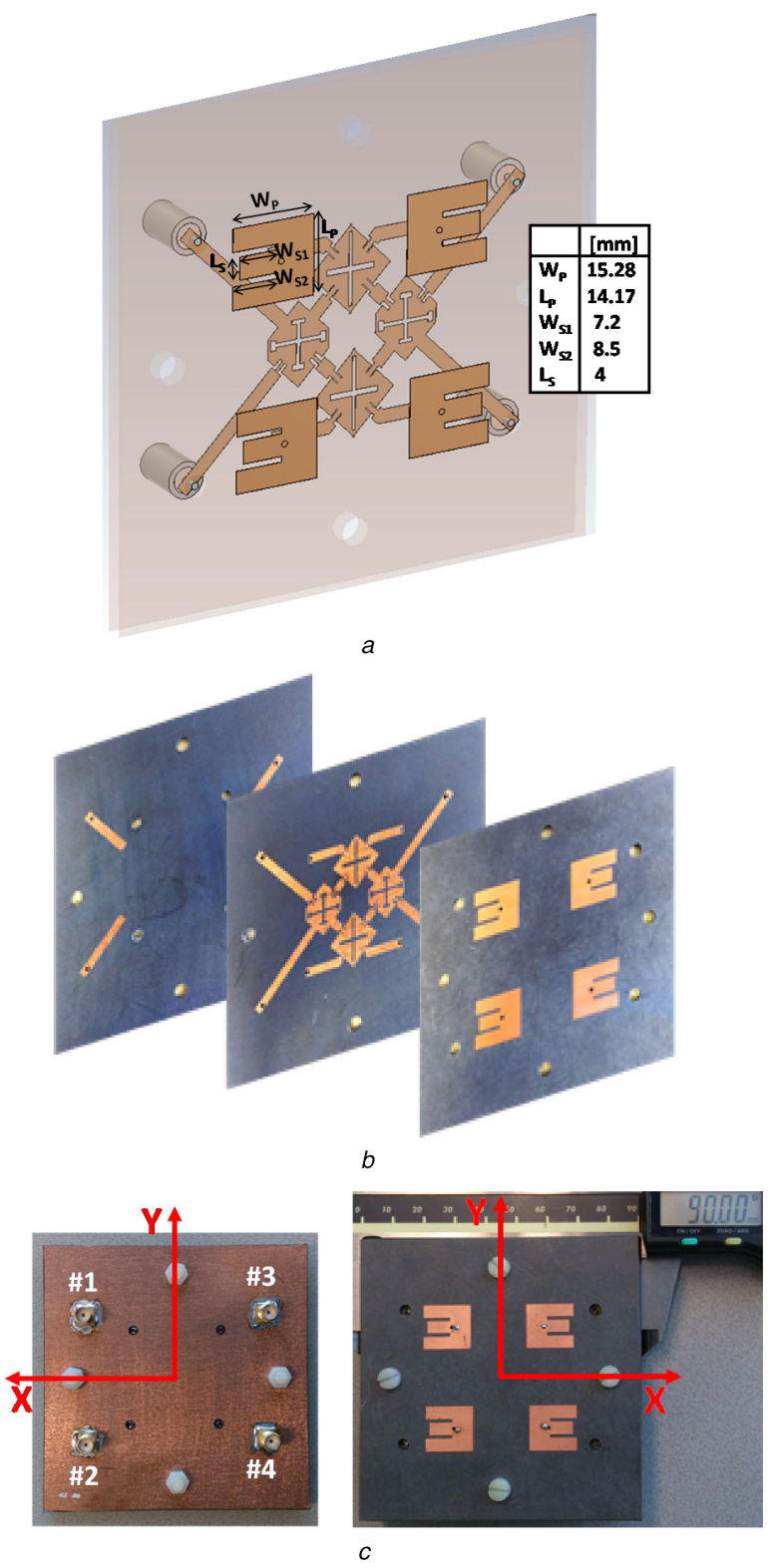

Fig. 9 The proposed subarray

(a) 3D view on the design, (b) Layers of the structure, (c) Assembled structure, back view, (d) Assembled structure, front view

measured results compare fairly well to simulations, at least in the operation band. There, discrepancies below the $-10 \mathrm{~dB}$ level are not larger than what could be expected from these benchmarking papers. Outside the operation band, the agreement gets worse, also above the $-10 \mathrm{~dB}$ level, see [26, 27].

Each input port is related to a desired beam direction. Ports $1-4$ are related to beams in the second, first, third and the fourth quadrants of the coordinate system, respectively. Input ports were driven sequentially while radiation patterns were measured in the 

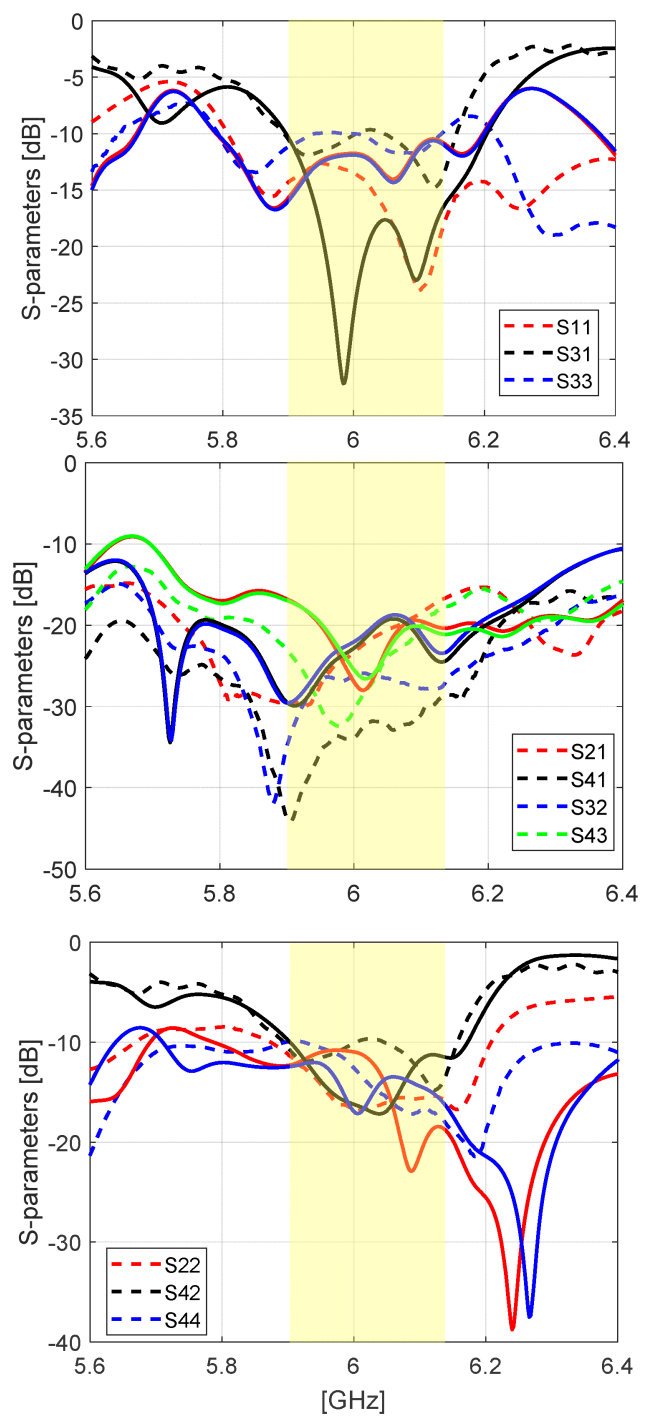

Fig. 10 S-parameters: measurement (dashed lines) and simulation (solid lines)

Table 3 Directions of beams

\begin{tabular}{lccc}
\hline Port & Theta & Phi & Directivity \\
\hline 1 & $19^{\circ}$ & $145^{\circ}$ & 11.7 \\
2 & $35^{\circ}$ & $40^{\circ}$ & 10.9 \\
3 & $35^{\circ}$ & $220^{\circ}$ & 10.9 \\
4 & $19^{\circ}$ & $325^{\circ}$ & 11.7 \\
\hline
\end{tabular}

Phi $=145^{\circ}, 40^{\circ}, 220^{\circ}$ and $325^{\circ}$ planes, respectively. Simulated and measured radiation patterns in two perpendicular planes are shown in Fig. 11, clearly displaying the directive lobes deviating from broadside. Measurements and simulations agree well with each other in terms of main beams widths and directions. The simulated directivities are $11 \mathrm{dBi}$, approximately, for all four beams, as shown in Table 3. The realised gain when port 1 is fed is given in Fig. 12. Table 4 compares the gain and scan angle range of the proposed subarray with some published 2D arrays driven by Butler matrices.

The idea was also very recently and partially discussed in a short paper [28]. However, it has been considerably expanded. Not only is the design now introduced in a very conceptual and systematic step-by-step way, but also much more detailed information is given by providing the full and detailed design of all modules.

\section{Conclusion}
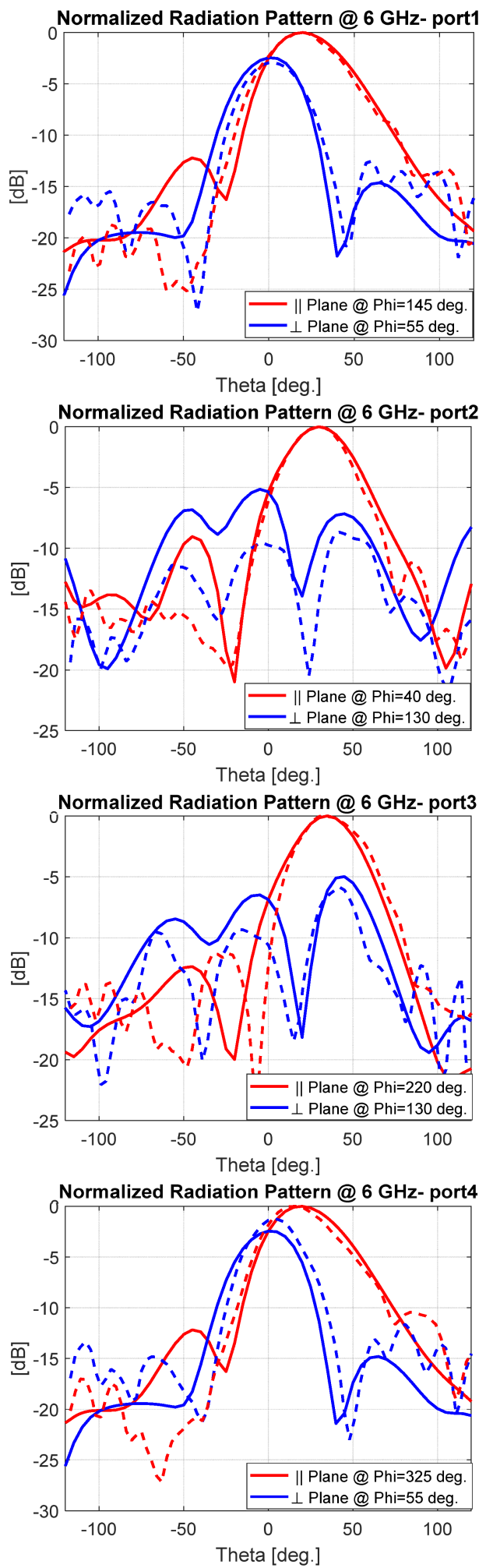

Fig. 11 Measured (dashed line) and simulated (solid line) radiation pattern cuts in main beam plane and its perpendicular plane

A multi-beam array antenna attachable to a typical wall and suitable for indoor ubiquitous wireless communications was designed. It is an example of a basic sub-array to realise the socalled 'e-wall' concept. The array has beam steering capabilities in $2 \mathrm{D}$, both elevation and azimuth planes. To achieve this goal, a novel stripline compact BFN was proposed which feeds four Eshaped patch antennas in a 2 by 2 arrangement. The proposed BFN is a very compact modified Butler matrix which consists of four stripline couplers. The design, simulation and measurement results have been presented. The resulting structure is small enough to be used as a building block for larger e-wall arrays. 


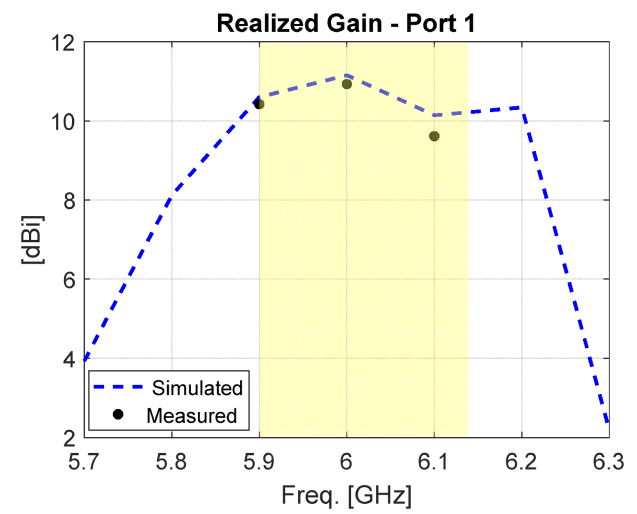

Fig. 12 Realised gain

Table 4 Comparison of 2D arrays driven by Butler matrices

\begin{tabular}{lcccc}
\hline Ref. & Can be used as building block in larger & $\begin{array}{c}\text { Measured peak gain, } \\
\text { array? }\end{array}$ & $\mathrm{dBi}$ & Theta scan range, deg. \\
\hline$[2]$ & no, too large $\left(2.9 \times 2.9 \lambda^{2}\right)$ & 12.9 & -38 to 38 & efficiency $69 \%$ \\
{$[13]$} & no, too large $\left(3 \times 3 \lambda^{2}\right)$ & 10.1 & -30 to 30 & - \\
{$[12]$} & yes & not reported & -30 to 30 & all ports are used to develop \\
& yes & 11 & -35 to 35 & - \\
this work & each beam & - \\
\hline
\end{tabular}

\section{Acknowledgment}

This project has received funding from the European Union's Horizon 2020 research and innovation program under the Marie Sklodowska-Curie grant agreement no. 721732.

\section{References}

[1] Larsson, E.G., Edfors, O., Tufvesson, F., et al.: 'Massive MIMO for next generation wireless systems', IEEE Commun. Mag., 2014, 52, (2), pp. 186195

[2] Guntupalli, A.B., Djerafi, T., Wu, K.: 'Two-dimensional scanning antenna array driven by integrated waveguide phase shifter', IEEE Trans. Antennas Propag., 2014, 62, (3), pp. 1117-1124

[3] Aerts, W., Delmotte, P., Vandenbosch, G.A.: 'Conceptual study of analog baseband beam forming: design and measurement of an eight-by-eight phased array', IEEE Trans. Antennas Propag., 2009, 57, (6), pp. 1667-1672

[4] Aliakbarian, H., Van der Westhuizen, E., Wiid, R., et al.: 'A digitally beamsteerable antenna array system for positioning-based tracking applications', IEEE Antennas Propag. Mag., 2013, 55, (6), pp. 35-49

[5] Xu, H., Aliakbarian, H., Vandenbosch, G.A.: 'Off-the-shelf low-cost target tracking architecture for wireless communications', IEEE Syst. J., 2015, 9, (1), pp. 13-21

[6] Moody, H.: 'The systematic design of the Butler matrix', IEEE Trans. Antennas Propag., 1964, 12, (6), pp. 786-788

[7] Ren, F., Hong, W., Wu, K.: 'W-band series-connected patches antenna for multibeam application based on SIW butler matrix'. 11th European Conf. on Antennas and Propagation (EUCAP), Paris, France, March 2017, pp. 198-201

[8] Wincza, K., Gruszczynski, S.: 'Broadband integrated $8 \times 8$ butler matrix utilizing quadrature couplers and Schiffman phase shifters for multibeam antennas with broadside beam', IEEE Trans. Microw. Theory Tech., 2016, 64, (8), pp. 2596-2604

[9] Yang, Q.L., Ban, Y.L., Lian, J.W., et al.: 'SIW butler matrix with modified hybrid coupler for slot antenna array', IEEE Access, 2016, 4, pp. 9561-9569

[10] Zhu, J., Peng, B., Li, S.: 'Cavity-backed high-gain switch beam antenna array for 60-GHz applications', IET Microw. Antennas Propag., 2017, 11, (12), pp. 1776-1781

[11] Chou, H.T., Yu, C.T.: 'Design of phased array antennas with beam switching capability in the near-field focus applications', IET Microw. Antennas Propag., 2015, 9, (11), pp. 1120-1127

[12] Slomian, I., Wincza, K., Gruszczynski, S.: 'Single-layer four-beam microstrip antenna array'. Radio and Wireless Symp. (RWS), Phoenix, AZ, USA, January 2017, pp. 29-31

[13] Karamzadeh, S., Rafii, V., Kartal, M., et al.: 'Modified circularly polarised beam steering array antenna by utilised broadband coupler and $4 \times 4$ butler matrix’, IET Microw. Antennas Propag., 2015, 9, (9), pp. 975-981
[14] Tran, H.H., Park, I.: 'Wideband circularly polarized $2 \times 2$ antenna array with multibeam steerable capability', IEEE Antennas Wirel. Propag. Lett., 2017, 16, pp. 345-348

[15] Tian, G., Yang, J.P., Wu, W.: 'A novel compact Butler matrix without phase shifter', IEEE Microw. Wirel. Comput. Lett., 2014, 24, (5), pp. 306-308

[16] Dall'Omo, C., Monediere, T., Jecko, B, et al.: 'Design and realization of a $4 \times$ 4 microstrip Butler matrix without any crossing in millimeter waves', Microw. Opt. Tech. Lett., 2003, 38, (6), pp. 462-465

[17] Nedil, M., Denidni, T.A., Talbi, L.: 'Novel Butler matrix using CPW multilayer technology', IEEE Trans. Microw. Theory Tech., 2006, 54, (1), pp 499-507

[18] Djerafi, T., Wu, K.: 'A low-cost wideband 77-GHz planar Butler matrix in SIW technology', IEEE Trans. Antennas Propag., 2012, 60, (10), pp. 4949 4954

[19] Cheng, Y.J., Hong, W., Wu, K.: 'Millimeter-wave multibeam antenna based on eight-port hybrid', IEEE Microw. Wirel.Comput. Lett., 2009, 19, (4), pp. 212-214

[20] Zheng, S., Chan, W.S., Leung, S.H., et al.: 'Broadband butler matrix with flat coupling', Electron. Lett., 2007, 43, (10), pp. 576-577

[21] Rao, P.H., Sajin, J.S., Kudesia, K.: 'Miniaturisation of switched beam array antenna using phase delay properties of CSRR-loaded transmission line', IET Microw. Antennas Propag., 2018, 12, (12), pp. 1960-1966

[22] Sun, S., Zhu, L.: 'Miniaturised patch hybrid couplers using asymmetrically loaded cross slots', IET. Microw. Antennas Propag., 2010, 4, (9), pp. $1427-$ 1433

[23] Gandini, E., Ettorre, M., Sauleau, R., et al.: 'A lumped-element unit cell for beam-forming networks and its application to a miniaturized butler matrix', IEEE Trans. Microw. Theory Tech., 2013, 61, (4), pp. 1477-1487

[24] Jeong, Y.S., Kim, T.W.: 'Design and analysis of swapped port coupler and its application in a miniaturized Butler matrix', IEEE Trans. Microw. Theory Tech., 2010, 58, (4), pp. 764-770

[25] Ang, B.K., Chung, B.K.: 'A wideband E-shaped microstrip patch antenna for 5-6 GHz wireless communications', Prog. Electromagn. Res., 2007, 75, pp. 397-407

[26] Vasylchenko, A., Schols, Y., De Raedt, W., et al.: 'Quality assessment of computational techniques and software tools for planar antenna analysis', IEEE Antennas Propag. Mag., 2009, 51, (1), pp. 23-38

[27] Vandenbosch, G.A.E.: 'State-of-the-art in antenna software benchmarking: 'Are we there yet?", IEEE Antennas Propag. Mag., 2014, 56, (4), pp. 300 308

[28] Salarrahimi, M., Vandenbosch, G.A.E.: 'Compact butler network for 2Dsteered array'. 12th European Conf. on Antennas and Propagation (EUCAP), London, UK, April 2018 